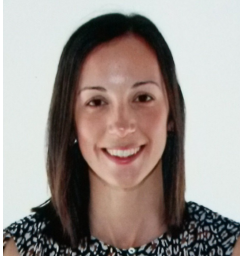

\title{
Introducción a la localización de sitios web optimizados para dispositivos móviles
}

Cristina Plaza Lara

Universidad de Málaga

\section{RESUMEN}

Las páginas web con diseño optimizado para su correcta visualización en dispositivos móviles han supuesto nuevos desafíos en el proceso de localización. En este artículo, repasamos algunas de las singularidades de este proceso, tanto desde la fase de internacionalización como la de traducción, testeo y gestión de proyectos.

Palabras clave: dispositivos móviles, localización, páginas web de diseño optimizado, RDW

\section{ABSTRACT}

Web page designs optimised so that they can be displayed on mobile devices properly have posed new challenges for localisation. This article reviews some of the peculiarities of this process at the different stages - internationalisation, translation and testing phase as well as project management.

Keywords: mobile devices; localisation; optimised web page design; RDW

\section{RESUM}

Les pàgines web amb disseny optimitzat per a la correcta visualització en dispositius mòbils han suposat nous reptes en el procés de localització. En aquest article repassem algunes de les singularitats d'aquest procés, tant des de la fase d'internacionalització com des de la fase de traducció, verificació i gestió de projectes.

Paraules clau: dispositius mòbils, localització, pàgines web de disseny optimitzat, RDW

\section{Introducción}

Hoy en día nadie duda de que la revolución tecnológica que se produjo a finales del siglo pasado modificó el escenario de trabajo de la traducción profesional. No solo se desarrollaron nuevos procesos de trabajo, sino que también encontramos nuevos productos que requerían de la traducción para su correcto funcionamiento en mercados de todo el mundo. Los dispositivos móviles han vuelto a abrir una nueva brecha tecnológica y las tabletas y los teléfonos inteligentes se han convertido en herramientas de nuestro día a día que nos permiten estar conectados y buscar información en cualquier lugar y en cualquier momento.

Aunque no podemos limitar la definición de dispositivos móviles a tabletas y smartphones, es cierto que estos dos tipos han sido los que mayor impacto han tenido hasta el momento en nuestras vidas diarias. Todo ello repercute directamente en la forma de trabajo de millones de profesionales $\mathrm{y}$, en el caso de la traducción, supone también una transformación de los tipos de textos que se traducen. La localización de dispositivos y aplicaciones móviles se ha convertido en un nuevo campo de especialización y estos contenidos han empezado a impartirse en másteres (véanse los distintos másteres en tradumática que se ofrecen en el ámbito español) y cursos de especialización de traducción.

Teniendo presente el contexto que acabamos de describir, el objetivo de este artículo es abordar la localización de sitios web optimizados o adaptados a dispositivos móviles (en 
inglés conocidos como responsive design websites, RDW). El responsive web design se puede definir de la siguiente forma:

RWD basically allows a website to respond or adapt to a different viewport size, smaller or larger, without your having to set a specific domain/subdomain for people using mobile devices. The look and feel of the website can be maintained as to have similar experiences across different device sizes. This is possible with the use of viewport meta tag and CSS3 media queries. (Firdaus, 2013: 8)

Aunque en este artículo no abordaremos el diseño de este tipo de páginas web, para quienes deseen profundizar en el tema citaremos a tres autores que, en nuestra opinión, abordan este concepto de manera clara para no expertos: Firdaus (2013), Fielding (2014) y Peterson (2014).

Según datos del Consumer Barometer de Google (https://www.consumerbarometer.com/en/), el $31 \%$ de los usuarios que más accede a Internet lo hace solo a través de su smartphone. Ante esta situación, Google decidió adaptar sus algoritmos con un atributo de posicionamiento orgánico, según el cual para aquellas búsquedas realizadas desde dispositivos móviles se indexarán primero las páginas adaptadas a estos dispositivos. Al margen de estas cuestiones técnicas (que obviamente favorecen la creación de sitios web adaptados al mundo móvil), como usuarios habituales de dispositivos móviles podemos afirmar que aquellas páginas que no están adaptadas dificultan en exceso la navegación. Pongamos como ejemplo la página web de un periódico con la vista clásica y la vista adaptada a móviles:

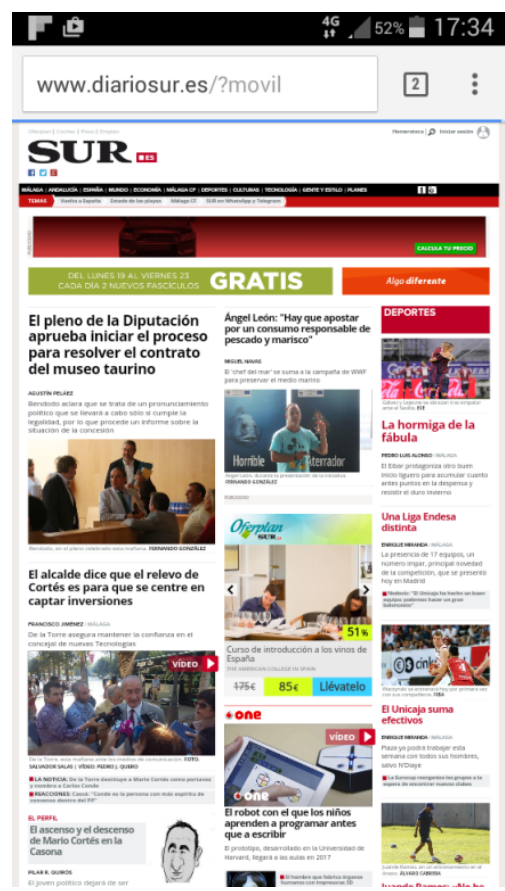

Figura 1. Página del Diario Sur (http://www.diariosur.es/) en la vista clásica

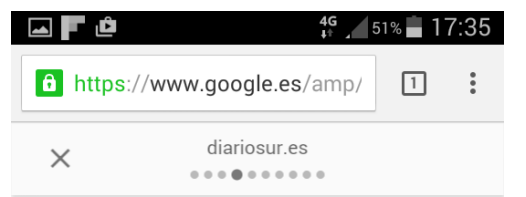

\section{SUR⿴囗十}

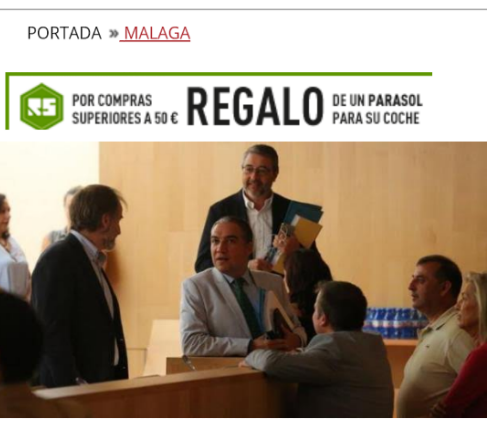

El pleno de la Diputación aprueba con el voto en contra del PP iniciar el proceso para resolver el

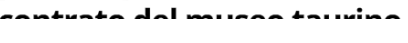

Figura 2. Página del Diario Sur (http://www.diariosur.es/) en la vista para móviles

Para navegar y leer en la primera vista es necesario hacer zoom. En la segunda vista, se ha adaptado la página al tamaño de la pantalla y facilita su lectura. Estas dos imágenes solo vienen a confirmar que la adaptación de los sitios web a los dispositivos móviles se ha convertido en un requisito fundamental para facilitar la navegación y que los usuarios permanezcan más tiempo en nuestra página. 


\section{El proceso de localización de RDW para dispositivos móviles}

En este apartado abordaremos algunas de las peculiaridades que la localización de sitios web con diseño optimizado para móviles presenta con respecto a la localización de páginas web diseñadas para su visualización y consulta en dispositivos de mayor tamaño. Aunque se siguen cumpliendo algunos rasgos y muchas de estas tareas de adaptación dependen del equipo de desarrollo del sitio web, nos encontramos con nuevos desafíos técnicos, lingüísticos y de gestión. Dichos desafíos se presentarán según las fases de trabajo de un proyecto de localización.

\subsection{Internacionalización}

Dentro del conocido modelo GILT (siglas para globalización, internacionalización, localización y traducción), desarrollado por la LISA (Localisation Industry Standards Association), la internacionalización se definió como:

Internationalization is the process of generalizing a product so that it can handle multiple languages and cultural conventions without the need for re-design. Internationalization takes place at the level of program design and document development. (LISA, cit. en Esselink, 2000: 2)

Aunque esta asociación desapareció en 2011 y podemos encontrar otras definiciones más completas (por ejemplo, Pym 2004; Jiménez Crespo, 2008; Fernández Costales, 2010), los pilares básicos sobre los que se asienta esta definición siguen siendo válidos. Si la extrapolamos al ámbito de las RDW, además de aquellas cuestiones habituales como la extracción del texto o la codificación de caracteres, encontramos otras como la disposición espacial del sitio web.

El principio estructural del que parten las RDW es el uso de porcentajes para establecer las medidas de las distintas partes de un sitio web. Así, en lugar de establecer medidas fijas, se les asigna una proporción que permitirá su adaptación a pantallas de menor tamaño. En la siguiente imagen ejemplificamos de forma clara este concepto:

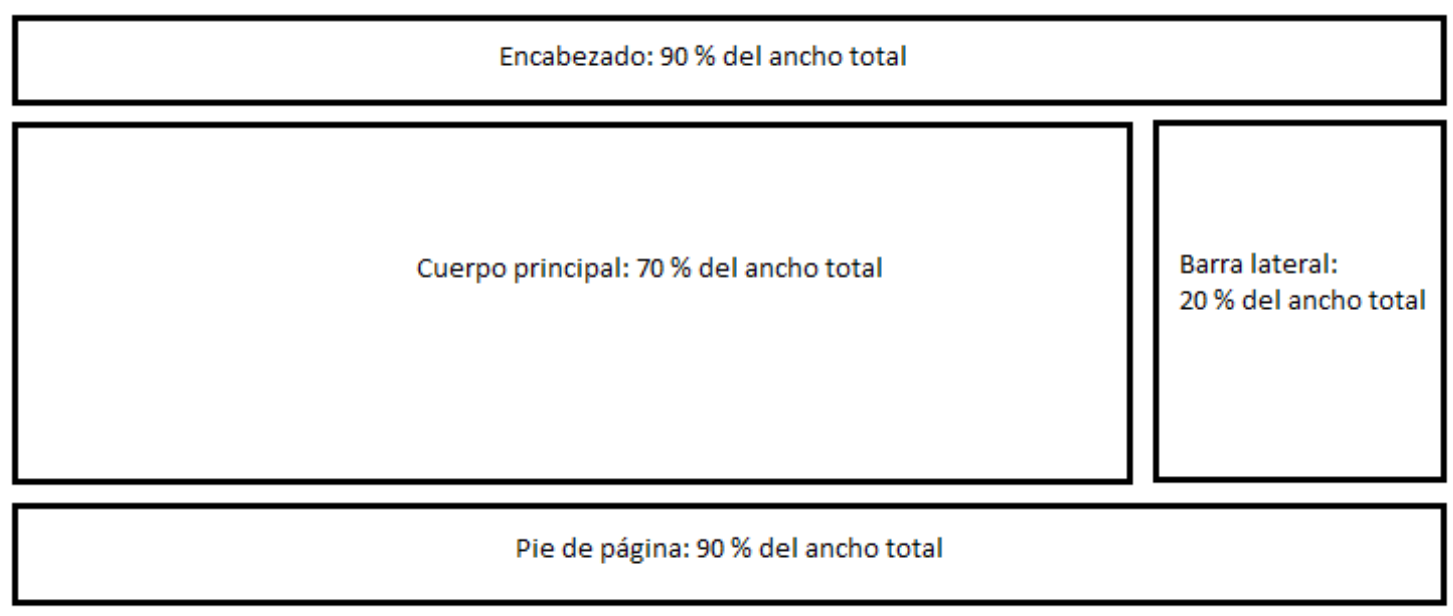

Figura 3. Fluid design o fluid layout en el diseño web adaptativo

Este diseño no supone un problema para la localización de RDW, sino más bien una solución a los problemas de espacio que se encontraban en la localización web tradicional, ya que la página web se adaptará a la longitud del texto y de la pantalla, independientemente del idioma en el que esté escrito. El diseño gira en torno al texto y no al contrario.

Otro aspecto importante que tener en cuenta es el uso de los llamados breakpoints o puntos de interrupción. Estos no son más que aquellos puntos en los que se introduce una división en una página o un elemento para ajustarse al tamaño de la pantalla. Tal y como se 
explica en la página de la University College London (https://www.ucl.ac.uk/indigo/designfoundation/macro-micro-breakpoints), existen dos tipos: los macroestructurales, que son aquellos aplicables al diseño global de la página, y los microestructurales, para ajustes menores de texto. Así pues, durante la fase de internacionalización, sería interesante realizar una pseudotraducción (usando las funciones que ofrecen los programas de traducción asistida), con el fin de que el desarrollador web pueda introducir estos puntos en el diseño en función de la longitud del idioma. Asimismo será imprescindible la colaboración entre el desarrollador y el equipo de traducción, para añadir breakpoints microestructurales en determinadas partes de la página que por la longitud del texto requieran una distribución diferente según el idioma.

Por último, con respecto a la extracción del texto, tenemos que diferenciar entre páginas web en HTML y páginas web diseñadas con un CMS (Content Management System). Las primeras agrupan la mayoría de investigaciones sobre RWD, ya que en el caso de los CMS se suelen utilizar plantillas con un diseño adaptativo que facilitan en parte la tarea. Así pues, para extraer el texto de una página web en HTML simplemente tendremos que recurrir a los archivos con esta extensión. Sin embargo, en el caso de los CMS, que separan el diseño del contenido, trabajaremos principalmente con un archivo po y algunos plugins del propio sistema.

\subsection{Localización}

En lo que respecta a la fase de localización, vamos a dejar a un lado cuestiones aplicables a la localización de todo tipo de página web (colores, referencias culturales, imágenes, etc. [véase, por ejemplo, Jiménez Crespo, 2008; Pym, 2010]) y nos centraremos en los contenidos. Partimos de la premisa de que disponemos de un texto original que debemos traducir fielmente. Sin embargo, nos encontramos con una serie de limitaciones en su mayoría espaciales, a las que podríamos añadir otras más recientes y no exclusivas de la localización de RDW, como el uso de palabras clave para la optimización SEO.

Algunas de las definiciones tradicionales de localización ya indicaban que localización y traducción no son lo mismo (véase, por ejemplo, Fernández Costales, 2010: 51, o Plaza Lara, 2014: 24), si limitábamos la traducción a su definición más tradicional. La transcreación e incluso el copywriting se han convertido en tareas que empiezan a desempeñar los propios traductores y, en el caso de las RDW, dadas las limitaciones de espacio que supone leer en una pantalla pequeña, se están incluso definiendo pautas para la redacción de contenidos. EI responsive content se puede definir como:

[...] a way of making the content of our site adapt or morph based on the behavioral context of the user.

The most obvious example of this in practice is displaying different layouts, information or features for users of mobile devices compared to users of desktop machines. (Sharkie y Fisher, 2013)

Mencionamos aquí algunos puntos interesantes que también deberían tener en cuenta los traductores de este tipo de páginas, y no solo los redactores. La página web de Google Developers (https://developers.google.com/web/fundamentals/design-and-ui/content/?hl=en) incluye algunos de estos consejos:

Puesto que la mayoría de las personas que visitan una web escanean su contenido antes de leerlo, se debe utilizar un lenguaje sencillo.

La idea principal debe estar reflejada en los primeros párrafos, de forma que sea fácil de identificar por el usuario de la web.

Una postura minimalista tanto de diseño como de contenidos permite que la información sea localizable por el lector, por lo que se deben eliminar los contenidos 
innecesarios y repetitivos y se deben emplear títulos para localizar la información de un vistazo.

Se sugiere que en una columna de texto cada línea debe contener entre 70 u 80 caracteres, por lo que el uso de los breakpoints se hace imprescindible. Al respecto de los caracteres, debemos remitirnos también al concepto responsive typography que no es otra cosa que el uso de caracteres que se adapten a la pantalla mediante diferentes técnicas (Harb et al., 2011: 21).

Hoy en día es posible encontrar multitud de blogs en los que se aborda el tema de la redacción de contenidos web e incluso en la bibliografía especializada en RDW se incluyen capítulos específicos de desarrollo de contenidos (véase, por ejemplo, de León, 2016: 23 o Peterson, 2014: 19). Al margen de estas recomendaciones de redacción, no podemos olvidar que se están desarrollando algunas herramientas o plugins para adaptar el texto al tamaño de la pantalla, bien omitiendo parte de él o modificándolo ligeramente. Como ejemplo mencionaremos Sifter App (https://sifterapp.com/) o el plugin SO Responsive content (https://es.wordpress.org/plugins/so-visibility-classes/), aunque en este campo queda aún mucho por explorar.

\subsection{Testeo}

La multitud de dispositivos que tenemos hoy en día al alcance de la mano obliga a los desarrolladores a probar sus aplicaciones y páginas web en distintos sistemas operativos y navegadores. Sería inviable económicamente que para dicha comprobación las empresas tuvieran que adquirir los distintos dispositivos. Para este fin, se han creado máquinas virtuales y servicios de testeo que permiten comprobar una página web adaptada a diferentes tamaños de pantalla. Aunque estas herramientas están en constante evolución, mencionamos solo algunas de ellas para evitar que el contenido que aquí ofrecemos se quede obsoleto: BrowSync (https://www.browsersync.io/), Ghostlab (https://www.vanamco.com/ghostlab/), Adobe Inspect (http://www.adobe.com/es/products/edge-inspect.html), Remote Preview (https://viljamis.com/2012/remote-preview/),

BrowserStack

(https://www.browserstack.com/start), entre otros muchos.

En el caso de que el traductor quiera visualizar el texto en pantallas más pequeñas durante la traducción y no en la fase de testeo, existen páginas como Responsivepx (http://responsivepx.com/) que permiten ver en línea una página web adaptada a unas dimensiones concretas. Asimismo encontramos iniciativas que facilitan el acceso a los dispositivos físicos durante un periodo de tiempo para que se pueda realizar el testing (véase, por ejemplo, Open Device Lab [https://opendevicelab.com/] o Labcase [http://labcase.org/]).

\subsection{Gestión de proyectos}

El proceso de localización de sitios web con diseño optimizado para móviles supone la creación de web multilingües, que requieren a su vez que cada uno de los idiomas cuente con su propia página optimizada. Esto podría implicar una multiplicación innecesaria de procesos (tanto lingüísticos como técnicos) si durante la gestión del proyecto no se toman las decisiones adecuadas de manera conjunta con el equipo de desarrollo. Podemos mencionar dos formas de conseguir un RDW:

En el caso de que tengamos ya un sitio web, rediseñarlo con el fin de optimizarlo para dispositivos móviles, de forma que tendríamos el sitio web para ordenadores y el sitio web para dispositivos móviles.

Si no tenemos web, podemos desarrollarla desde cero con diseño optimizado. Además, se pueden convertir páginas no optimizadas a RDW y evitar de esta forma duplicar el sitio web. 
En el primer caso tendríamos el sitio web de escritorio y de móviles más sus distintas versiones en diferentes idiomas. En el segundo caso, solo existirían RDW multilingües. Se puede concluir que la segunda opción sería la más viable en cuestión de rentabilidad, ya que no implicaría repetir procesos de manera innecesaria para las distintas versiones. Desde el punto de vista técnico, resulta más sencillo adaptar un sitio web a dispositivos de mayor tamaño que al contrario. Desde una perspectiva lingüística, también supondría la gestión de memorias de traducción, glosarios y referencias para cada versión.

Por otro lado, y en relación con la definición tradicional de localización, en el caso de las RDW se hace imprescindible también gestionar los contenidos para adaptarlos al público de la cultura de destino. Estos procesos de arquitectura de la información se complican aún más si a ello le añadimos limitaciones de espacio. Así pues, el gestor de proyectos deberá contar con un completo equipo de adaptación y gestión de contenidos que sea capaz de ajustar el sitio web a las necesidades del usuario final.

\section{Conclusiones}

Los dispositivos móviles han venido a modificar la forma de desarrollar sitios web así como los contenidos de cada uno de ellos. La web dinámica es hoy en día una realidad y los usuarios están habituados a interactuar de forma activa con esta información. El contenido para localizar ya no se encuentra siempre en archivos estáticos, sino en bases de datos que permiten visualizar la información contenida en ellas.

El escenario global en el que nos movemos ha acelerado la aparición de sitios web multilingües y las imposiciones técnicas de los grandes motores de búsqueda obligan a las empresas a adaptarse si quieren aumentar su presencia en la Red. En el caso de las RDW, Google por ejemplo indexa primero aquellas páginas con contenido optimizado, siempre que la búsqueda se realice desde un dispositivo móvil. Esto ha provocado que, tal y como ocurrió en los inicios de la localización, las competencias tecnológicas y estratégicas de los traductores se vean reforzadas si quieren ofrecer a sus clientes un servicio integral. Se precisan de conocimientos técnicos para la extracción del texto y adaptación a distintos dispositivos, de gestión de contenidos y de proyectos de gran envergadura, de diseño web y asesoramiento lingüístico y cultural para que el cliente obtenga un producto completo en diferentes idiomas. Se hace imprescindible, por tanto, el trabajo conjunto del equipo de localización y desarrollo web. Sería interesante, por ejemplo, la creación de guías de estilo, no solo lingüísticas, sino también de diseño web, en las que se especifique la estructura global del sitio en diferentes idiomas y en diferentes dispositivos, si procede, así como el uso de fuentes, colores, enlaces, ubicación de menús y botones, entre otras muchas cuestiones.

En conclusión, tal y como hemos expuesto en este artículo, además de las aplicaciones, la localización de sitios web optimizados para dispositivos móviles ha supuesto cambios en el proceso de localización en su totalidad, incorporando nuevos procedimientos y nuevas herramientas que pretenden facilitar en la medida de lo posible la labor del equipo lingüístico y de diseño. 


\section{Bibliografía}

Consumer Barometer with Google. <https://www.consumerbarometer.com/en/> Datos de consulta de la página: 26.09.2016.

De León, I. (2016). Moving to Responsive Web Design. Bring Existing Static Sites into Today's Multi-Device World with Responsive Web Design. Londres: Apress.

Diario Sur <http://www.diariosur.es/> Datos de consulta de la página: 31.10.2016.

Esselink, B. (2000). A practical guide to localization. Ámsterdam, Filadelfia: John Benjamins.

Fernández Costales, A. (2010). Traducción, localización e internacionalización. El caso de las páginas web universitarias. Tesis doctoral. Oviedo: Universidad de Oviedo.

Fielding, J. (2014). Beginning Responsive Web Design with HTML5 and CSS3. Nueva York: Apress.

Firdaus, T. (2013). Responsive Web Design by Example. Olton, GB: Packt Publishing.

Google Developers. Multi-device content. <https://developers.google.com/web/fundamentals/design-and-ui/content/?hl=en> Datos de la última actualización: 06.12.2011. Datos de consulta de la página: 26.09.2016.

Harb, E. et al. (2011). Responsive Web Design.

<http://courses.iicm.tugraz.at/iaweb/surveys/ws2011/g3-survey-resp-web-design.pdf> Datos de consulta de la página: 26.09.2016.

Jiménez Crespo, M. A. (2008). El proceso de localización web: estudio contrastivo de un corpus comparable del género sitio web corporativo. Tesis doctoral. Granada: Universidad de Granada.

Peterson, C. (2014). Learning Responsive Web Design. A Beginner's Guide. Canadá: O'Reilly.

Plaza Lara, C. (2014). La enseñanza de la traducción asistida por ordenador: análisis didáctico-curricular y propuesta de integración de la subcompetencia instrumentalprofesional en el aula. Tesis doctoral. Málaga: Universidad de Málaga.

Pym, A. (2004). The moving text. Ámsterdam, Filadelfia: John Benjamins.

Pym, A. (2010). Website localization <http://usuaris.tinet.cat/apym/online/translation/2009_website_localization_feb.pdf> Datos de la última actualización: 28.01.2010. Datos de consulta de la página: 31.10.2016.

Sharkie, C. y Fisher, A. (2013). Jump Start Responsive Web Design. Australia: Sitepoint.

University Collage London. Macro and micro breakpoints. <https://www.ucl.ac.uk/indigo/design-foundation/macro-micro-breakpoints> Datos de consulta de la página: 26.09.2016. 